\title{
Fellows Column: Unexpected Pneumomediastinum with Spontaneous Resolution in a Newborn
}

Rebekah Frazier, MSIV, Shabih Manzar, MD

\begin{abstract}
Spontaneous pneumomediastinum is uncommon in neonates. We report a case of unexpected pneumomediastinum with spontaneous resolution in a newborn supported by a literature review.
\end{abstract}

\section{Keywords:}

Pneumomediastinum, Respiratory distress, Spontaneous Resolution, Newborn, cesarean

\section{"Pneumomediastinum, also known \\ as mediastinal emphysema, can \\ be a complication of respiratory \\ distress. While most cases of neonatal \\ pneumomediastinum result in \\ spontaneous resolution, there is a risk \\ of pneumothorax, subcutaneous, and \\ interstitial emphysema with deterioration \\ of the clinical status $(3,4,5)$. Therefore, treatment and close observation are \\ warranted."}

\section{Introduction:}

Respiratory distress is defined as any sign of breathing difficulty in a newborn. This could present as tachycardia, grunting, cyanosis, or subcostal/intercostal retractions $(1,2)$. Common causes of neonatal respiratory distress include transient tachypnea of the newborn, respiratory distress syndrome, pneumonia, sepsis, meconium aspiration syndrome, pneumothorax, pulmonary hypertension, cardiac failure, and hypoxic-ischemic encephalopathy.
Pneumomediastinum, also known as mediastinal emphysema, can be a complication of respiratory distress. While most cases of neonatal pneumomediastinum result in spontaneous resolution, there is a risk of pneumothorax, subcutaneous, and interstitial emphysema with deterioration of the clinical status $(3,4,5)$. Therefore, treatment and close observation are warranted.

\section{Case Presentation:}

A male infant was delivered via cesarian section at 39 weeks 5 days gestation $\left(39^{5 / 7}\right)$ to a Gravida 1 , Para 0, Term 0, Preterm 0 , Abortion 0, Living 0 (G1P0000) female. Pregnancy was complicated by fetal cardiac echogenic focus (Quad screen, NIPT neg, confirmed Level II) and group B streptococcus infection. Mother was admitted for induction of labor and progressed to complete dilation. The infant was unable to be delivered despite vacuum assistance. Delivery progressed to a low-transverse c section with epidural anesthesia. At birth, the infant weighed 3485 grams and presented with Apgar scores of 8 and 9 at one and five minutes. The infant required continuous positive airway pressure (CPAP) in the delivery room. On arrival to the Neonatal intensive care unit (NICU), the infant was placed on the bubble CPAP. The infant remained critically ill on bubble CPAP with $\mathrm{FiO} 2$ 21-40\%. Chest $X$-ray was concerning for pneumomediastinum/pneumopericardium and questionable right anterior pneumothorax (Figures $1 \mathrm{~A}$ and 1B). Pneumopericardium was ruled out due to hemodynamic stability and the absence of halo around the cardiac silhouette. Transillumination was performed at bedside negative for pneumothorax. The echocardiogram was within normal limits. Oral nutrition was started on day 1 of life with 20 cc Similac ProAdvance every 3 hours, gradually increasing to full oral feeds.

On admission to the NICU, the infant's vital signs were temperature $98.1^{\circ} \mathrm{F}$, heart rate 172 beats/min, mean blood pressure 83/65 $\mathrm{mmHg}$. Initial blood glucose was $79 \mathrm{mg} / \mathrm{dL}$. Arterial blood gas showed $\mathrm{pH} 7.27, \mathrm{pCO}_{2} 43.4, \mathrm{pO}_{2} 179, \mathrm{HCO}_{3}$ 19.1. The respiratory exam was significant for subcostal retractions, tachypnea, and grunting with spontaneous respiratory effort. The abdominal exam was insignificant with a flat abdomen, normal bowel sounds in all four quadrants, and no organomegaly.

On day 2 of life, the infant was comfortable with grunting, and retractions improved. Follow-up chest X-ray showed stable pneumomediastinum (Figure 1C) with the patient on room air. The infant was
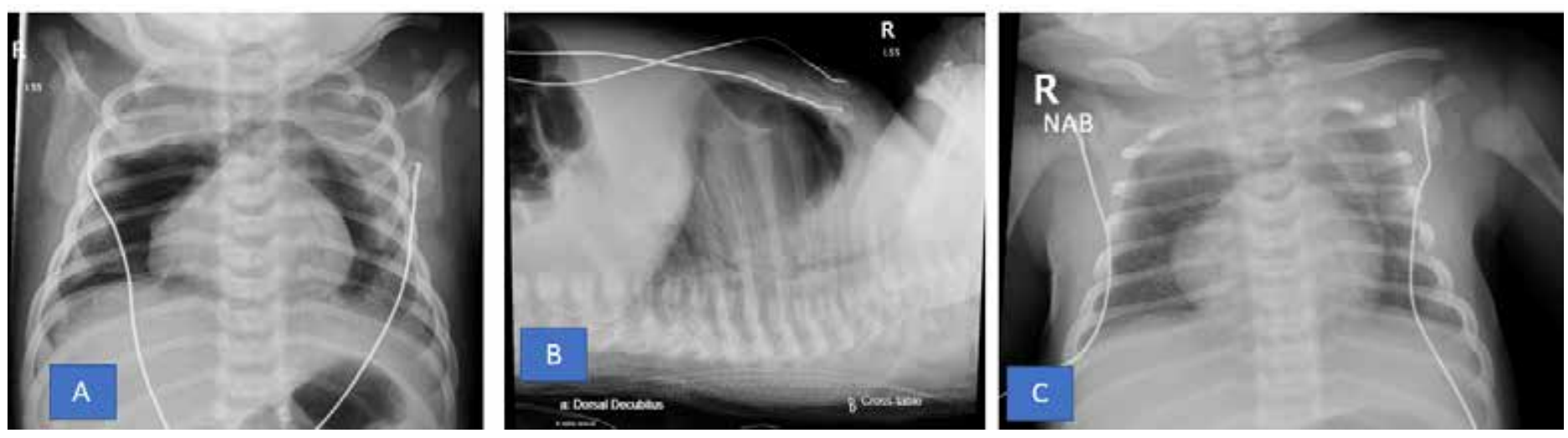

Figure 1 A: Chest x-ray, AP view, with significant pneumomediastinum; Figure $1 \mathrm{~B}$ : Chest x-ray, cross-table lateral view, with significant anterior pneumomediastinum; Figure $1 \mathrm{C}$ : Chest $x$-ray, AP view, with significant resolution of pneumomediastinum 
discharged home and last seen in the clinic on day 5 of life in stable condition with no residual respiratory distress and gaining weight.

\section{Discussion:}

Neonatal pneumomediastinum occurs in approximately 2.5 per 1000 live births (8).

A study performed in Zurich, Switzerland, showed that the incidence of pneumomediastinum (PM) was $0.1 \%$ of ICU neonates (4).

Pneumomediastinum in neonates is associated with prematurity, pneumonia, meconium aspiration, difficult delivery, and the need for positive pressure during resuscitation or mechanical ventilation (5). Furthermore, respiratory difficulties that may predispose to pneumomediastinum can occur due to cesarean delivery (6). We can infer from this information that this infant's difficult delivery (unsuccessful vacuum assistance and progression to c section) and need for CPAP during resuscitation may have led to PM findings.

\section{"Diagnosis of PM is first confirmed by chest X-ray, which may show the Spinnaker-Sail sign (7). PM treatment in the neonate is based primarily on symptoms and predominantly includes respiratory support such as oxygen therapy and CPAP (9)."}

Diagnosis of PM is first confirmed by chest $X$-ray, which may show the Spinnaker-Sail sign (7). PM treatment in the neonate is based primarily on symptoms and predominantly includes respiratory support such as oxygen therapy and CPAP (9).

\section{Conclusions:}

In conclusion, while often asymptomatic, infants with respiratory distress should be evaluated for pneumomediastinum with X-ray and treated with respiratory support. Follow-up x-rays should be obtained to evaluate for spontaneous resolution.

\section{References:}

1. Edwards, Martin $O$ et al. "Respiratory distress of the term newborn infant." Paediatric respiratory reviews vol. 14,1 (2013): 29-36; quiz 36-7. doi:10.1016/j.prrv.2012.02.002

2. Hermansen CL, Mahajan A. Newborn Respiratory Distress. Am Fam Physician. 2015;92(11):994-1002.

3. Raissaki M, Modatsou E, Hatzidaki E. Spontaneous pneumomediastinum in A term newborn: atypical radiographic and ct appearances. BJR Case Rep. 2019;5(4):20180081. Published 2019 Nov 15. doi:10.1259/bjrcr.20180081

4. Hauri-Hohl A, Baenziger O, Frey B. Pneumomediastinum in the neonatal and paediatric intensive care unit. Eur J Pediatr. 2008;167(4):415-418. doi:10.1007/s00431-007-0517-9

5. Rocha G, Guimarães $H$. Spontaneous pneumomediastinum in a term neonate - case report. Clin Case Rep. 2017;6(2):314316. Published 2017 Dec 22. doi:10.1002/ccr3.1352

6. Zanardo V, Simbi AK, Franzoi M, Soldà G, Salvadori A, Trevisanuto $D$. Neonatal respiratory morbidity risk and mode of delivery at term: influence of timing of elective caesarean delivery. Acta Paediatr. 2004;93(5):643-647. doi:10.1111/j.1651-2227.2004.tb02990.x

7. Monteiro R, Paulos L, Agro Jd, Winckler L. Neonatal spontaneous pneumomediastinum and the Spinnaker-Sail sign. Einstein (Sao Paulo). 2015;13(4):642-643. doi:10.1590/ S1679-45082015A/3133
8. Hacking $D$, Stewart M. Images in clinical medicine. Neonatal pneumomediastinum. N Engl J Med 2001; 344:1839.

9. Corsini I, Dani C. Clinical management of the neonatal pneumomediastinum. Acta Biomed. 2014;85(1):39-41. Published 2014 Jun 20.

Conflicts of Interest: The authors have no conflicts of interest relevant to this article to disclose.

Financial Disclosure: The authors have no financial relationships relevant to this article to disclose.

NT
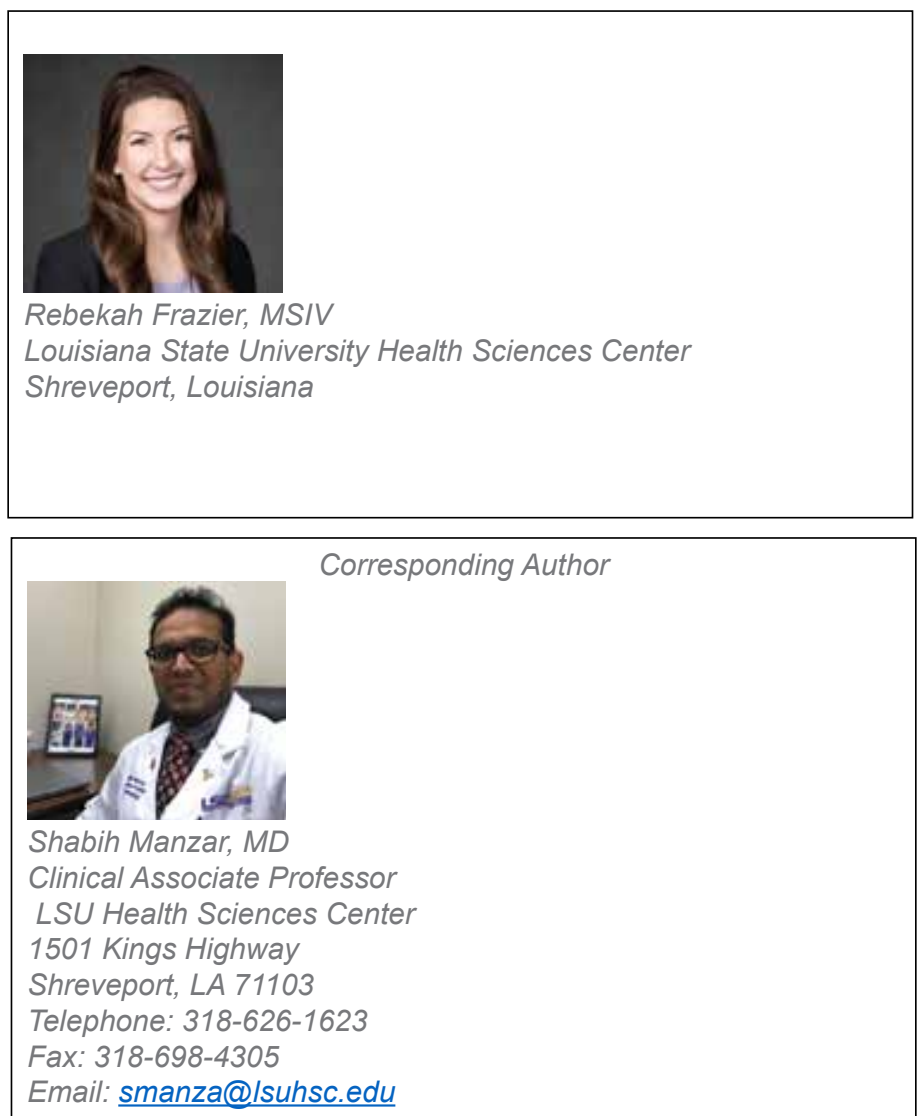

Fellow's Column is published monthly.

- Submission guidelines for "Fellow's Column":

- 2000 word limit not including references or title page. Exceptions will be made on a case by case basis

- $\quad$ QI/QA work, case studies, or a poster from a scientific meeting may be submitted..

- Submission should be from a medical student, resident, fellow, or NNP in training.

- Topics may include Perinatology, Neonatology, and Younger Pediatric patients.

- No more than 20 references.

- Please send your submissions to:

Elba Fayard, MD, Interim Fellowship Column Editor or Japmeet Sandhu, OMS III, Fellowship Column Assistant Editor LomaLindaPublishingCompany@gmail.com 